\title{
Open Challenges in SLAM: An Optimal Solution Based on Shift and Rotation Invariants
}

\author{
Agostino Martinelli, Nicola Tomatis and Roland Siegwart \\ Swiss Federal Institute of Technology Lausanne (EPFL) \\ CH-1015 Lausanne, Switzerland \\ e-mail: agostino.martinelli, nicola.tomatis, roland.siegwart depfl.ch
}

\begin{abstract}
This paper starts with a discussion of the open challenges in the SLAM problem. In our opinion they can be grouped in two main and distinct areas: convergence of the built map and computation requirement for real world application. To deal with the previous problems, a solution in the stochastic map framework based on the concept of the relative map is proposed. The idea consists in introducing a map state, which only contains quantities invariant under shift and rotation and to carry out the estimation of this relative map in an optimal way. This is a possible way in order to have a decoupling between the robot motion and the landmark estimation and therefore not to rely the landmark estimation on the unmodeled error sources of the robot motion. Moreover, the proposed solution scales linearly with the number of landmark allowing real-time application. Experimental results, carried out on a real platform, show the better performance of this method with respect to the joint vehicle-landmark approach (absolute map filter) when the odometry is affected by undetected systematic errors or by large or unmodeled non-systematic errors.
\end{abstract}

\section{INTRODUCTION}

In the Simultaneous Localization and Mapping (SLAM) problem a mobile robot has to be able to autonomously explore the environment with its on-board sensors, gain knowledge about it, interpret the scene, build an appropriate map and localize itself relative to this map.

Many approaches have been proposed to solve the SLAM problem both in the framework of the metric and the topological navigation.

A very successful method is the stochastic map approach. After the first precise mathematical definition of the stochastic map [15] early experiments ([4], [8]), have shown the quality of fully metric simultaneous localization and map building: the resulting environment model permits highly precise localization that is only bounded by the quality of the sensor data. However, these approaches suffer from some limitations. Firstly, they rely strongly on odometry. For automatic mapping this makes the global consistency of the map difficult to maintain in large environments where the drift in the odometry becomes too important. Furthermore, they represent the robot position with a single Gaussian distribution. This means that an unmodeled event (i.e. collision) could cause divergence between the ground truth and the estimated position from which the system is unable to recover (lost situation). In [1] it has been shown that by taking into account all the correlations the global consistency is better maintained. However, this is not sufficient as confirmed by another work [2] where a solution is proposed by extending the absolute localization to include a localization relative to local reference frames.

Currently the SLAM has two contrasting problems to be solved, which are often faced with a trade-off:

- The map convergence;

- The computational requirement (both in processing and memory) for real-time/real-world implementation

The aim of this paper is to suggest a solution in the frame-work of the stochastic map approach to SLAM, able to satisfy both the previous requirements. The basic idea consists in introducing a map state which only contains quantities invariant under shift and rotation. This is a possible way in order to have a decoupling between the odometry and the landmark estimation and therefore not to rely the landmark estimation on the unmodeled error sources in the robot motion. Only the case of point landmark is here considered although the same idea could be applied to other kind of landmark. In section II we discuss the two open challenges by illustrating the solutions so far adopted. In particular, the approach here introduced is compared with the previous filters based on the relative maps. The proposed filter is presented in section III for the case of point landmark. The results obtained through real experiments are displayed in section IV where also a comparison with the absolute map filter is shown. Finally, conclusions and future research are given in section $\mathrm{V}$.

\section{Open Challenges in SLAM}

\section{A. The Convergence Problem}

In order to find a solution of the SLAM problem it is clearly necessary to known the statistical model characterizing the error of each robot's sensor as better as possible. Only in this case it is possible to integrate all the information coming from the sensor of the robot in a proper manner. A statistical model for a sensor reading concerns the characterization of both the systematic and the non-systematic components of the error of the sensor. When the gaussian model is adopted to characterize the error of a given sensor, the systematic component is provided by the mean value and the non-systematic component by the variance. Since the previous quantities (mean value and variance) are known only with a finite accuracy and since the gaussian hypothesis is in general a simplifying approximation, any approach to SLAM based on this error model will produce a divergence in the built map if the environment is large 
enough. This problem arises even if the approach is optimal respect to the dynamics of the robot and the observation and if the convergence is theoretically proven. Indeed, the divergence arises because the error model is unperfect.

In [7], the convergence of a filter which estimates the robot configuration and the absolute location of the landmarks by adopting a Kalman filter (absolute map filter, $A M F$ ), is theoretically proven. However, the proof is based on a perfect statistical knowledge of the error of each sensor and also on the hypothesis of a linear observation. The map convergence is not proven when the hypothesis of linearity is not fully satisfied (see [10] for theoretical details).

In order to minimize the divergence of the built map, one have to concentrate on two important points:

- Adopt an optimal filter (accordingly to the dynamics and the observation);

- Use the best statistical model to characterize the error of the adopted sensor readings (it is better avoiding the use of sensors whose error is roughly known (statistically) in the estimation process)

Clearly, to deal with the second remark, it is better not to use the odometry in the estimation phase if, as often happens, other more precise sensors are available with a well-known error model. The AMF, using odometry, diverges when there is even a very small, undetected systematic component. In [10] we proved the divergence through simulation while here this divergence is proven through experiments on a real platform. Moreover, both in [10] and here in section IV, we do not consider completely unmodeled events (e.g. collisions) which cause an error in the odometry estimation very far from the assumed odometry error model. Therefore, decoupling odometry from the estimation process is very important. Csorba, Uhlmann and Durrant-Whyte [5] introduced a relative map based on quantities invariant to the robot pose (i.e. to shift and rotation). The same idea was adopted by Deans and Hebert [6]. Both estimate the distance between two landmarks, which is invariant to the robot pose (shift and rotation). However, their algorithms are sub optimal because they do not consider any correlation between the distances. In figure 1 we display an observation consisting of 4 landmarks. Let consider the distance $d_{12}$ between the first and the second landmark. Clearly, the error on this distance get from this observation is correlated with the error on $d_{13}, d_{14}, d_{23}$ and $d_{24}$ and it is not correlated with the error on $d_{34}$. In other words, the covariance matrix for the vector $\left[d_{12}, d_{13}, d_{14}, d_{23}, d_{24}, d_{34}\right]$ will be:

$$
\left[\begin{array}{cccccc}
\sigma_{d_{12}}^{2} & \sigma_{d_{12} d_{13}} & \sigma_{d_{12} d_{14}} & \sigma_{d_{12} d_{23}} & \sigma_{d_{12} d_{24}} & 0 \\
\sigma_{d_{12} d_{13}} & \sigma_{d_{13}}^{2} & \sigma_{d_{13} d_{14}} & \sigma_{d_{13} d_{23}} & 0 & \sigma_{d_{13} d_{34}} \\
\sigma_{d_{12} d_{14}} & \sigma_{d_{13} d_{14}} & \sigma_{d_{14}}^{2} & 0 & \sigma_{d_{14} d_{24}} & \sigma_{d_{14} d_{34}} \\
\sigma_{d_{12} d_{23}} & \sigma_{d_{13} d_{23}} & 0 & \sigma_{d_{23}}^{2} & \sigma_{d_{23} d_{24}} & \sigma_{d_{23} d_{34}} \\
\sigma_{d_{12} d_{24}} & 0 & \sigma_{d_{14} d_{24}} & \sigma_{d_{23} d_{24}} & \sigma_{d_{24}}^{2} & \sigma_{d_{24} d_{34}} \\
0 & \sigma_{d_{13} d_{34}} & \sigma_{d_{14} d_{34}} & \sigma_{d_{23} d_{34}} & \sigma_{d_{24} d_{34}} & \sigma_{d_{34}}^{2}
\end{array}\right]
$$

The importance of these correlations arises in the estimation process. In the filter proposed by Deans and Hebert [6], the estimated distance at a given time step is a combination of the two estimations of that distance coming from the last

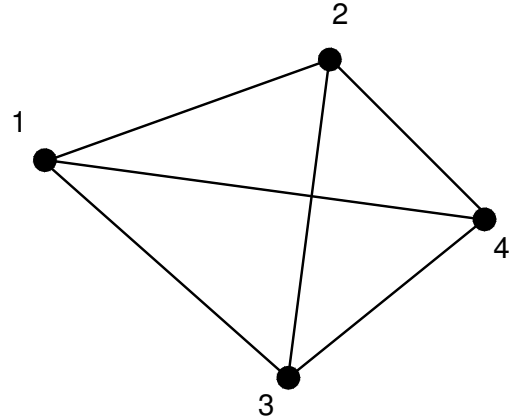

Fig. 1. The distance between the first and the second landmark is correlated with all the other distances except with the one between the third and fourth landmark

update and the current observation (see equation (10) in [6]). In our case, since we use a Kalman Filter which maintains the correlations, the estimation of a given distance is a combination of all the distances correlated with it. In particular, the estimation of a given distance can be updated even in the case when this distance is not re-observed. Indeed, taking into account these correlations means to take into account of all the constraints among the distances (which are not independent) between the landmarks observed at least once simultaneously [11]. In section III-A we give the equations characterizing our filter.

Newman introduced a relative map and he used two filters in the estimation, called the relative map filter and the geometric projection filter ([12] and [13]). The second one provides a means to produce a geometrically consistent map from the relative map, by solving a set of linear constraints (indeed, he introduced a relative state containing quantities that are not independent). Both filters are optimal since the dynamics and the observation are both linear and they are based on the Kalman Filter. However, the elements used in this approach are invariant for shift only, not for rotation. Our approach is to take invariant elements, but for both shift and rotation and to apply a KF for estimation, contrasting to [5] and [6], who use the same invariants in combination with a non-optimal filter. The observation, as well as the dynamic, will be linear. Therefore the filter will be optimal with respect to both the previously presented criteria. The only error source, which could create a divergence in the long term, is the gaussian assumption adopted in the statistical knowledge of the external sensor (laser in the experiments).

\section{B. Computational Requirement}

As explained, the other open challenge for the SLAM is a practical problem, which one encounters when implementing an approach: the memory and processing resources are limited. This is especially present in the AMF filter, since the lack of invariance requires taking into account all the covariances [1]. This means that an update needs the update of all the covariances between landmarks and between the landmarks and the robot (which scales as $O\left(N^{2}\right)$, where $N$ is the number of landmarks). Therefore, AMF scales dramatically 
with the number of landmarks making impossible a real-time implementation when $N$ is larger than about one hundred. Thrun [16] proposed to reduce this complexity by using the Information Filter, which allows to detect the dependencies between landmarks and to set them to 0 if they are very small (Sparsification). This approximation allows reducing the complexity, but avoids an optimal solution. He applied this method also in the case of multi-robots [17]. A constant time solution was proposed very recently by Newman [14] by considering several local maps. The relative maps proposed by Csorba et al. [5], Deans and Hebert [6] and Newman [12] scale linearly with $N$ since the covariance matrix is block diagonal. Also in our approach, the covariance matrix will be block diagonal without any approximation. Furthermore, even in the event of closing a loop, the relative map approaches present at least two advantages with respect to the $A M F$ :

- the elements of the relative state for the landmarks belonging to the same loop are uncorrelated (without approximation) before closing the loop;

- After closing a loop, only the elements of the relative state for the landmarks belonging to this loop will be correlated.

We can say that the relative map approaches minimize the number of the correlations necessary in order to completely use the information coming from closing a loop. The closing loop constrain can be integrated by updating only the independent elements in the relative state (i.e. by deriving the analytical dependency of the dependent elements on the ones independent), that is equivalent to the application of the Projection filter introduced by Newman [12].

\section{The Structure of the Relative Map Filter}

A possible way to decouple odometry from the estimation process is obtained by introducing a filter whose state only contains quantities invariant under shift and rotation. This is the idea characterizing the relative filter introduced here. Once the relative map has been estimated through this filter and the absolute location of a set of landmarks is known (e.g. by using the first observation) it is possible to build the absolute map. Therefore, the entire method contains two algorithms. The former estimates the relative map, the latter builds the absolute map. In the sections III-A and III-B respectively we describe the two algorithms.

\section{A. The Relative Map Filter}

The state estimated through this filter only contains the distances between the point landmarks. Of course, the distance is a quantity invariant under shift and rotation, i.e. it is independent of the robot configuration. Let denote with $d$ the state and with $P$ its covariance matrix. In fig. $2 a$ the vector $d$ contains the marked distances between the 6 landmarks. Clearly, not all of the distances between the 6 landmarks are stored in $d$ because not all the landmarks were observed together at the same time. At a given time step, the observation consists of a set of distances between the landmarks observed by the robot through its external sensor (fig. 2b). These

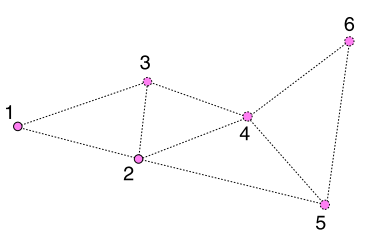

(a)

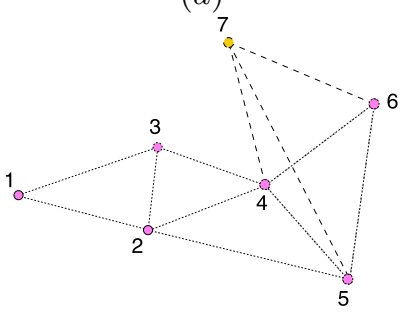

(c)

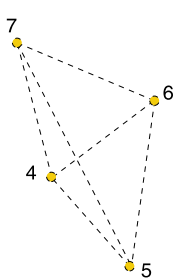

(b)
Fig. 2. Relative Map before the observation (a), the observation (b), and the relative map obtained by fusing the information coming from the old map and the observation (c). In all the three figures the map state only contains the indicated distances between the landmarks

distances may be already observed (i.e. can be in the vector d) or may not. Let introduce the following notation:

$$
d_{\text {old }}=\left[u, w_{\text {old }}\right]^{T} \quad d_{o b s}=\left[w_{o b s}, v\right]^{T}
$$

where $d_{\text {old }}$ is the state estimated at a given time step and $d_{o b s}$ is the observation at the same time step, containing a set of distances between the landmarks observed by the robot. $u$ contains the distances which are not re-observed (i.e. which do not appear in the vector $d_{o b s}$ ) and $w_{\text {old }}$ contains the distances re-observed (denoted by $w_{o b s}$ in the vector $d_{o b s}$ ). Finally, $v$ contains the distances observed for the first time at the considered time step. The covariance matrix of the previous vectors are:

$$
P_{o l d}=\left[\begin{array}{cc}
P_{u u} & P_{u w} \\
P_{u w}^{T} & P_{w w}
\end{array}\right] \quad P_{o b s}=\left[\begin{array}{cc}
R_{w w} & R_{w v} \\
R_{w v}^{T} & R_{v v}
\end{array}\right]
$$

We adopt the following notation to denote the estimated quantities, obtained by fusing the old state with the observed one (the new estimated distances are depicted in fig. $2 c$ ).

$$
\begin{gathered}
d_{\text {new }}=\left[u_{\text {new }}, w_{\text {new }}, v_{\text {new }}\right]^{T} \\
P_{\text {new }}=\left[\begin{array}{lll}
P n_{u u} & P n_{u w} & P n_{u v} \\
P n_{u w}^{T} & P n_{w w} & P n_{w v} \\
P n_{u v}^{T} & P n_{w v}^{T} & P n_{v v}
\end{array}\right]
\end{gathered}
$$

We obtain the new estimation for the state and its covariance matrix by applying the equations of the Kalman filter. Observe that the observation is linear in the state (is the identity) and therefore the Kalman filter is optimal.

$$
u_{n e w}=u+P_{u w}\left(P_{w w}+R_{w w}\right)^{-1}\left(w_{o b s}-w_{o l d}\right)
$$




$$
\begin{gathered}
w_{n e w}=w_{o l d}+P_{w w}\left(P_{w w}+R_{w w}\right)^{-1}\left(w_{o b s}-w_{o l d}\right) \\
v_{n e w}=v+R_{v w}\left(P_{w w}+R_{w w}\right)^{-1}\left(w_{o l d}-w_{o b s}\right) \\
P n_{u u}=P_{u u}-P_{u w}\left(P_{w w}+R_{w w}\right)^{-1} P_{w u} \\
P n_{u w}=P_{u w}-P_{u w}\left(P_{w w}+R_{w w}\right)^{-1} P_{w w} \\
P n_{u v}=0 \\
P n_{w w}=P_{w w}-P_{w w}\left(P_{w w}+R_{w w}\right)^{-1} P_{w w} \\
P n_{w v}=R_{w v}-R_{w w}\left(P_{w w}+R_{w w}\right)^{-1} R_{w v} \\
P n_{v v}=R_{v v}-R_{v w}\left(P_{w w}+R_{w w}\right)^{-1} R_{w v}
\end{gathered}
$$

Instead of the equations (6) and (11) it is possible to use the following equations:

$$
\begin{gathered}
w_{n e w}=w_{o b s}+R_{w w}\left(P_{w w}+R_{w w}\right)^{-1}\left(w_{o l d}-w_{o b s}\right) \\
P n_{w w}=R_{w w}-R_{w w}\left(P_{w w}+R_{w w}\right)^{-1} R_{w w}
\end{gathered}
$$

They are derived by observing the symmetry of the filter with respect to the change "observation" $\leftrightarrow$ "old state". Observe that the coincidence of the previous equations could be easily proven also by using the inversion lemma.

\section{B. Recovering the Absolute Landmark Location}

We adopt a simple linear method to recover the absolute landmark locations starting from the absolute location of three landmarks and the state estimated by the previous filter, which contains the distances between the landmarks. At a given time step the absolute locations of a set of landmarks are available (we assumed that the absolute coordinates of at least three landmarks are known at the beginning; these coordinates could be provided by the first observation). The aim is to estimate the location of a new landmark denoted by $j$ (see fig. 3). We extract from the previous set a subset containing three landmarks whose distance from the landmark $j$ is provided by the relative filter (we assume that the extraction of three landmarks is possible; if it is not possible the absolute location of the landmark $j$ cannot be provided through this method). Let denote the locations of these landmarks by $\left(x_{i}, y_{i}\right)$ and the distance between the landmark $j$ and the landmark $i$ of this subset by $d_{i}$. Clearly, we have for the landmark $i$

$$
d_{i}^{2}=\left(x_{j}-x_{i}\right)^{2}+\left(y_{j}-y_{i}\right)^{2} \quad i=1,2,3
$$

We therefore obtain a linear system in the unknowns $x_{j}$ and $y_{j}$ by considering the differences $d_{2}^{2}-d_{1}^{2}$ and $d_{3}^{2}-d_{1}^{2}$. By

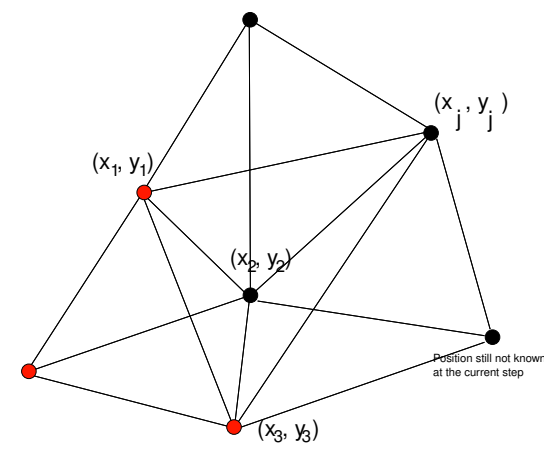

Fig. 3. In the estimation of the $j^{\text {th }}$ landmark's absolute location only the landmarks whose absolute position is known and whose distance from the $j^{\text {th }}$ landmark has been estimated through the relative filter are involved.

applying recursively this method for all the landmarks (all $j$ ) it is possible to get their absolute location at each time step.

Clearly, the choice of the three landmarks does not have any influence on the result since the distances are not independent and the dependency is automatically accounted by maintaining all the necessary correlations in the covariance matrix, as mentioned in the previous sections.

\section{EXPERIMENTS}

For the experiments, Donald Duck (see Fig. 4), a fully autonomous mobile robot, has been used. The robot is equipped with wheel encoders, two 180 laser range finders and a CCD camera (not used here). It is connected via radio ethernet only for data visualization via web and data logging for statistical purposes.

Ten beacons were placed in the environment. They could be detected by the laser sensor with an accuracy of about $2 \mathrm{~cm}$. They were adopted to create the point landmarks. Clearly, instead of them a function able to extract corners or any other point feature from the laser scanner could be used.

Figure 5 shows the metric map and the robot trajectory obtained through the absolute map filter. The odometry was calibrated through the method introduced in [9] before the experiment. By using this method of calibration it was possible to make the systematic odometry error smaller than $0.2 \%$. The robot moved along a closed trajectory at around $20 \mathrm{~cm} \mathrm{~s}^{-1}$ and estimated at each time step its configuration and the position of the beacons in the environment. In the figure, the estimated robot position is represented with a dot and the estimated beacon position with a circle. The unities are meters in both axes. The initial robot configuration coincides with the origin of the global reference whose axes were chosen coincident with the axis of the robot at the initial time. Because of the odometry calibration and the small dimension of the environment, the map convergence is good.

The next three figures (6-8) show the map and the robot trajectory as estimated by the AMF when a systematic error occurred (odometry not calibrated). They refer to the same experiment as in figure 5 but, in processing the data from the encoder sensor, the radius of the right wheel was changed by a factor equal to $0.5 \%, 1 \%$ and $2 \%$ respectively in the three 


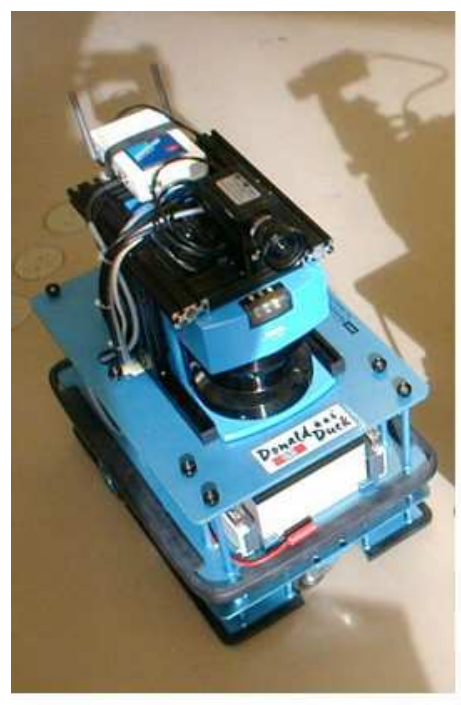

Fig. 4. The autonomous robot Donald Duck. Its controller consists of a VME standard backplane with a Motorola PowerPC 604 microprocessor clocked at 300 Mhz. Among its peripheral devices, the most important are the wheel encoders, a 360 laser range finder and a grey-level CCD camera (not used here).

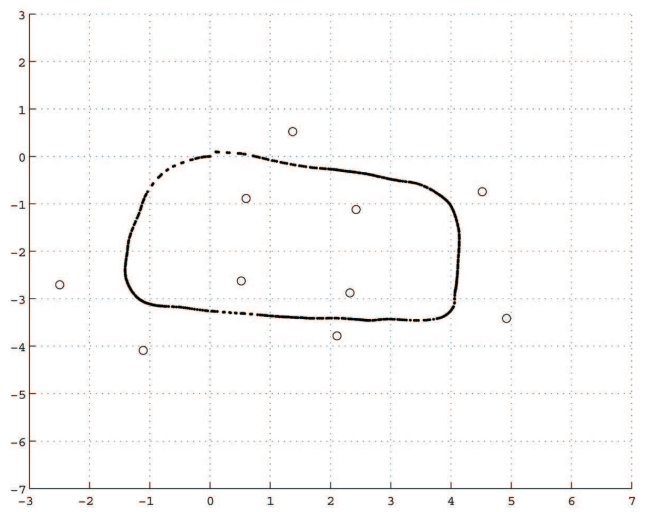

Fig. 5. The results obtained through the AMF when the odometry was perfectly calibrated $(<0.2 \%)$

figures. It is possible to see that the map starts to diverge even when the error is quite small $(0.5 \%)$. Moreover, when the error is larger or equal than $1 \%$ the algorithm makes an error in associating the data (in figure 7 the number of the beacons found are 12 and in figure 8 they are 16). Clearly, the error in the data association depends not only on the method adopted and on the sensor error, but also on the speed of the robot, on the frequency of the data delivery and on the actual distance among the landmarks. Therefore, the main problem here shown, concerns the map convergence.

Figure 9 shows the results obtained by using the same data adopted in the previous case, but through the relative map filter described in the section III. Since in this case the odometry was completely decoupled by the estimation process, there

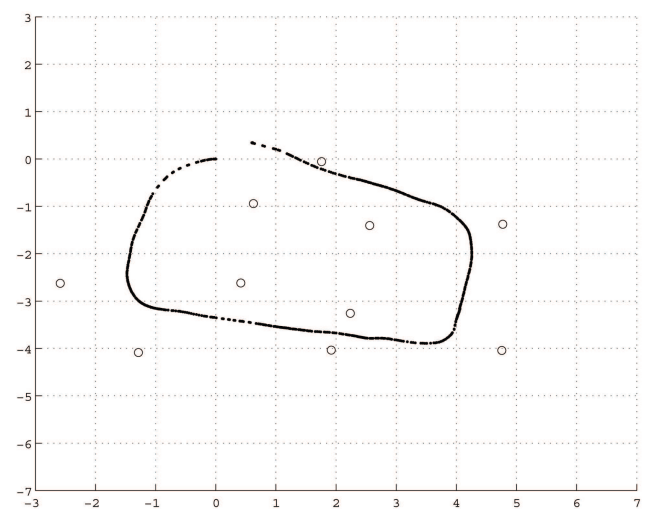

Fig. 6. The results obtained through the AMF when the radius of the right wheel was changed by a factor equal to $0.5 \%$

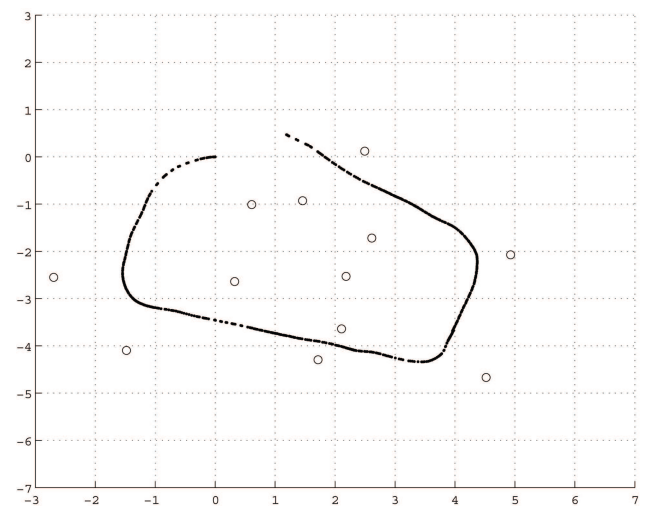

Fig. 7. The results obtained through the AMF when the radius of the right wheel was changed by a factor equal to $1 \%$

is not any drift in the built map. During the experiment the odometry data were only used to solve the data association problem and not in the estimation process. In this case, we get exactly the same result if we change the value of the wheel radius by a factor equal to $10 \%$. For changes larger an error in associating the data arises.

\section{Conclusions and Future Research}

This paper presented an approach to solve the SLAM problem in the stochastic map framework based on the concept of the relative map. The idea consists in introducing a map state which only contains quantities invariant under shifts and rotations and to carry out the estimation of this relative map in an optimal way (a Kalman filter was adopted). This is a possible way in order to have a decoupling between the robot and the landmark estimation and therefore not to rely the landmark estimation on the unmodeled error sources in the robot motion.

The optimal filter in combination with the invariance of the landmarks represents an excellent solution for the convergence. Furthermore, the filter presented here maintains all the covariances without approximation. On the other hand, the structure of the covariance matrix remains block diagonal due to the invariance. (Clearly, in the case of closing a loop all the 


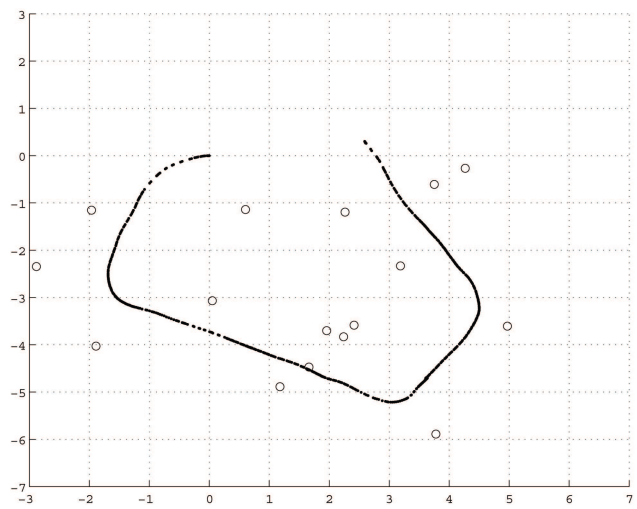

Fig. 8. The results obtained through the AMF when the radius of the right wheel was changed by a factor equal to $2 \%$

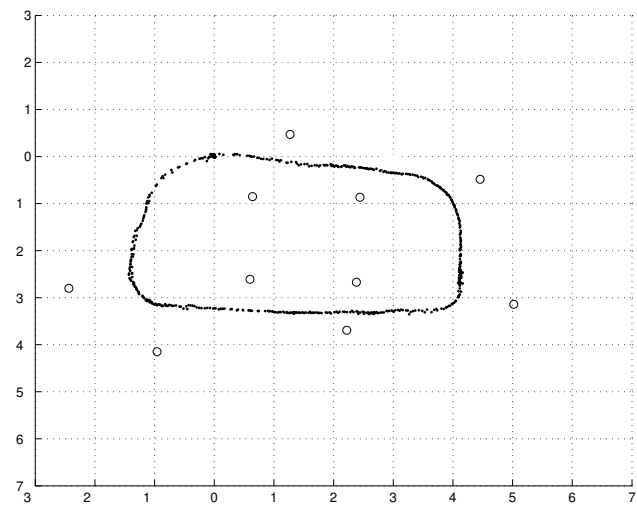

Fig. 9. The results obtained through the RMF. In this case the odometry was completely decoupled by the estimation process and therefore there is not any drift in the built map even when the wheel diameters is changed by a factor equal to $10 \%$

elements involved in the loop will be correlated, increasing the complexity of the covariance matrix. However, as for other relative map approaches, the number of correlations is the minimum necessary to completely use all the information coming from the closing loop constraint). Therefore, the presented method represents an optimal solution to the open challenges of SLAM pointed out at the beginning of the work: Convergence and computation. A series of experiments on a real platform was carried out to validate our method. In particular, the same data from the experiment were adopted to compare the standard joint vehicle-landmark approach (absolute map filter) with our method. We conclude that the convergence of the absolute map filter is true only if several infeasible hypothesis are satisfied (linear observation, odometry perfectly calibrated, non-systematic odometry error statistically perfectly known). The proposed approach does not require these hypothesis.

We are extending the approach to the case of more general landmarks. In particular, we are deriving the equations for this relative filter for the corner feature.

\section{Acknowledgments}

This work has been supported by the European project RECSYS (Real-Time Embedded Control of Mobile Systems with Distributed Sensing)

\section{REFERENCES}

[1] Castellanos, J. A., J. D. Tardos, et al. (1997). Building a Global Map of the Environment of a Mobile Robot: The Importance of Correlations. IEEE International Conference on Robotics and Automation (ICRA), Albuquerque.

[2] Castellanos, J. A., M. Devy, et al. (2000). Simultaneous Localization and Map Building for Mobile Robots: A Landmark-based Approach. IEEE International Conference on Robotics and Automation (ICRA), San Francisco.

[3] Chong K.S., Kleeman L., "Accurate Odometry and Error Modelling for a Mobile Robot," International Conference on Robotics and Automation, vol. 4, pp. 2783-2788, 1997.

[4] Crowley, J.L., (1989). World Modeling and Position Estimation for a Mobile Robot Using Ultrasonic Ranging. IEEE International Conference on Robotics and Automation (ICRA), Scottsdale, AZ.

[5] M.Csorba, J.K.Uhlmann and H.F.Durrant-Whyte"A subOptimal Algorithm For Automatic Map Building," American Control Conference, p. 537 -541, Albuquerque, New Mexico, USA 1997.

[6] M.C.Deans and M.Hebert, "Invariant Filtering for Simultaneous Localization and Mapping," International Conference on Robotics and Automation, vol. 2, pp. 1042 -1047, San Francisco, CA, USA, April 2000.

[7] Dissanayake, Newman, Clark, Durrant-Whyte and Csorba, 2001, A Solution to the Simultaneous Localization and Map Building (SLAM) problem, IEEE Trans. On Rob. And Aut. Vol 17, No.3, June 2001

[8] J.J. Leonard, H.F. Durrant-Whyte, "Directed Sonar Sensing for Mobile Robot Navigation," Kluwer Academic Publishers, Dordrecht, 1992.

[9] Martinelli A, Tomatis N, Tapus A. and Siegwart R., "Simultaneous Localization and Odometry Calibration" International Conference on Inteligent Robot and Systems (IROSO3) Las Vegas, USA

[10] Martinelli A and Siegwart R., "Improving the SLAM Convergence with a Relative Map Filter" submitted at the International Conference on Inteligent Autonomous Systems (IASO4) Amsterdam, The Netherlands.

[11] Martinelli A, Tomatis N and Siegwart R., "A Relative Map Approach to SLAM Based on Shift and Rotation Invariants" in preparation.

[12] P.M.Newman, "On the Structure and Solution of the Simultaneous Localization and Mapping Problem," PhD thesis, Australian Centre for Field Robotics, University of Sydney, 1999.

[13] P.M.Newman and H.F.Durrant-Whyte, "An efficent solution to the slam problem using geometric projection," Sensor Fusion and Decentralized Control in Robotics Systems, Boston, USA, 2001.

[14] P.M.Newman, J.J. Leonard and R.J.Rikoski, "Towards Constant-Time SLAM on an Autonomous Underwater Vehicle Using Syntheic Aperture Sonar,' International Symposyum of Robotics Research (ISRR03), October 2003, Siena, Italy.

[15] Smith, Self, et al. (1988) "Estimating uncertain spatial relationships in robotics" Uncertainty in Artificial Intelligence 2 Elsevier Science Pub: 435-461.

[16] S.Thrun, D.Koller, Z.Ghahramani, H.F. Durrant-Whyte and A.Y.Ng., "Simultaneous mapping and Localization with Sparse Extended Information Filters," Proceedings of the 5th Int. Workshop on Algorithmic Foundations of Robotics, Nice, France, 2002.

[17] S.Thrun and Y.Liu, "Multi-Robot SLAM with Sparse Extended Information Filters," International Symposyum of Robotics Research (ISRR03), October 2003, Siena, Italy. 